\title{
The Research of Buck Converter Sliding Mode Algorithm based on Power Function Exponential Reaching Law
}

\author{
Hongyang Liang ${ }^{1}$, Yongheng Li ${ }^{2,{ }^{a} *}$, Yiwei Liu ${ }^{3}$, Hong Li $^{4}$, Zhe Sun ${ }^{5}$ \\ ${ }^{1}$ Shenyang Military Logistics, Shenyang 110000 , China \\ ${ }^{2}$ the Aviation University of Air Force, Changchun 130022, China \\ ${ }^{3}$ The Represent Office of the PLA in SAC, Shenyang 110000, China \\ ${ }^{4}$ Meihekou Garden, Meihekou 135000, China \\ ${ }^{5} 95926$ Military, Changchun 130000, China \\ a870137364@qq.com
}

Keywords: buck converter, the power function exponential reaching law, chattering.

\begin{abstract}
The article designs a new kind of power function exponential reaching law based on exponential reaching law. It associates the speed of the trajectory and the distance of velocity to the sliding mode surface and uses intelligence features of the exponential function. The reaching law suppresses the chattering phenomenon and overcomes the exponential reaching law through a switch sliding mode surface. The power function exponential reaching law is applied to the Buck converter, and presents a specific Matlab/Simulink module, the simulation verified the effectiveness of this method.
\end{abstract}

\section{Introduction}

Sliding mode control is a kind of variable structure control method, it is not sensitive to the internal parameters and external disturbance, and has good robustness. Dynamic response is relatively excellent, suitable for engineering practice. Under ideal conditions, the switching frequency is infinite and won't produce the chattering. But in the real cases, chattering is unable to avoid. Chattering can cause high frequency oscillation in the system, it also put forward a great challenge for its application in the engineering practice.

Scholars at home and abroad focus on a lot of problem of chattering on the sliding mode control method.

Our scientists founded near rate method to reduce the chattering in the sliding mode movement problems and got the ideal effect ${ }^{[1]}$. The literature [2] proposed variable speed reaching law for the first time. It produced the fan switch area, gived the fan switch area and the mathematical model of quasi sliding mode. The literature [3] combined fuzzy control with sliding mode control, adjusted the coefficient of exponential reaching law through the fuzzy rules. It settled the input fuzzy rules to the absolute value $|s|$ of the switch function, the output of the fuzzy rules for coefficient and the exponential reaching law $\varepsilon$ and $k$, which could further improve the dynamic performance of the sliding mode, reduced the high frequency vibration of the system. The literature [4] designed a kind of adaptive variable speed reaching law in front of the track to sliding mode, used the adaptive exponential reaching law. It reached the sliding mode surface and used variable speed reaching law, effectively reduced the chattering. The literature [5] was based on power frequency approach Rate and integral reaching law. It designed a number of power points near rate and power frequency near rate index of integral sliding mode observer. The simulation results showed that the sliding mode observer had played a very good effect on inhibition of jitter. The literature [6] proposed a double exponential reaching law and increased the convergence speed of the system, the system had better dynamic quality. The literature [7] proposed a new discrete reaching law for the uncertain part of the system. Disturbance predictor was designed and made the system stable in the origin, had very high estimation precision, wreaked the chattering effectively. The literature [8] used fuzzy control for 
on-line estimation in view of the uncertainties in the system, fuzzed adaptive adjustment of the switch gain and reduced the switch gain as far as possible, in order to reduce chattering.

This paper designs a new kind of sliding mode reaching law the exponential function on the second order the Buck converter, the reaching law is based on sliding mode variable index reaching law. It adapts the design of the exponential function of symbols instead of sliding mode variable structure control switch function and a larger scale has the effect of adaptive adjustment, effectively suppress the chattering phenomenon.

\section{The mathematical model of Buck converter}

Buck converter is a DC Buck converter. It is also known as DC chopper, it is a kind of output voltage which is less than or equal to the average of the input voltage of the switch tube DC voltage converter.

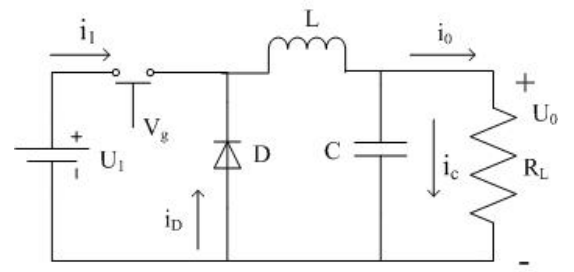

Fig.1 Buck converter topology structure diagram

Fig. 1 is Buck converter topology structure. $U_{1}$ stands for input DC voltage, $V_{g}$ stands for switch tube which is responsible for the entire circuit on and off, D stands for the fly-wheel diode, L, C stands for inductance and capacitance, $\mathrm{R}_{\mathrm{L}}$ stands for the load resistance.

When the switch tube $\mathrm{V}_{\mathrm{g}}$ conducts, charges current through the inductor $\mathrm{L}$ load; when switch tube Vg shuts off, the load current through the inductor L discharge. Buck converter maintains the stability of the load voltage by rapid on-off switch tube. Keeping the switch tube Vg on and off cycle can adjust the size of the load on both ends of the output current and voltage.

If load is the impedance load, we consider $i_{L}$ inductor current and capacitor voltage $\mathrm{v}_{\mathrm{C}}$ as state variables, ignore the inductance and capacitance of the parasitic resistance. We can get the state space equation of the Buck converter:

$$
\left[\begin{array}{c}
\frac{d v_{c}}{d t} \\
\frac{d i_{L}}{d t}
\end{array}\right]=\left[\begin{array}{cc}
-\frac{1}{C R_{L}} & \frac{1}{C} \\
-\frac{1}{L} & 0
\end{array}\right]\left[\begin{array}{c}
v_{c} \\
i_{L}
\end{array}\right]+\left[\begin{array}{c}
0 \\
\frac{v_{\mathrm{i}}}{L}
\end{array}\right] u
$$

$\mathrm{u}$ stands for state variables. When $\mathrm{u}=1$, corresponding switch tube $\mathrm{Vg}$ close. When $\mathrm{u}=0$, corresponding switch tube Vg open.

\section{The exponential function exponential reaching law}

Sliding mode variable structure control system produce the chattering because of system state point in the high speed switch. The existence of the switching function in the traditional switching surface symbols will inevitably bring system chattering, but if the system cancel the switching function, it will lose the definition of a variable structure. You can't have the sliding movement and lose the system robustness. To this end, I design a new type of switching function based on the exponential function instead of the traditional symbol switching function.

The switching exponential function compared with the traditional symbolic function and saturation function, it has adaptive adjustment effect in a larger scale. When $s$ is large, the system move point away from the switching surface $s=0, f a l(s, \alpha, \delta)>1$. The farther away from the switching surface, the greater function of fal modulus value; when $\mathrm{s}$ is small, system move and travel through the switching surface. The exponential function of switching compared with the traditional symbolic function and saturated function without severe switching, it is a relatively smooth transition curve. 


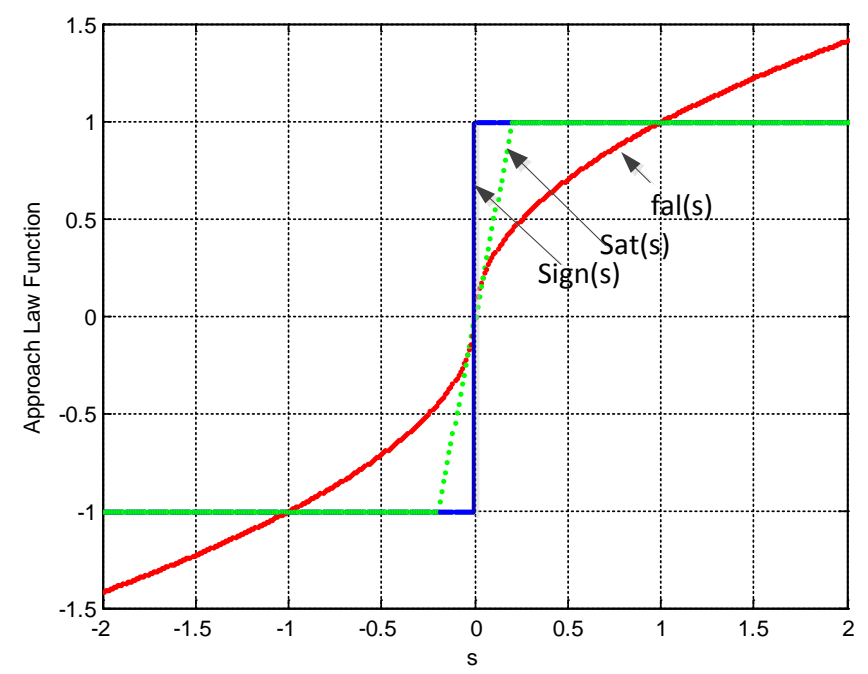

Fig.2 Comparison of three kinds of switching functions

According to the exponential function design of the new index of the sliding mode reaching law:

$$
\left\{\begin{array}{c}
\dot{s}=-\varepsilon \text { fal }(\mathrm{s})-\mathrm{ks} \\
\operatorname{fal}(\mathrm{s}, \alpha, \delta)=\left\{\begin{array}{c}
\frac{\mathrm{s}}{\delta^{1-\alpha}},|s|<\delta \\
|s|^{\alpha} \operatorname{sign}(\mathrm{s}),|s| \geq \delta
\end{array}\right.
\end{array}\right.
$$

Among them, $0<\alpha<1,0<\delta<1$.

Based on the lyapunov stability theory, list lyapunov stability function as follows:

$V(\mathrm{x})=\frac{s^{2}}{2}$

According to the formula (2) available:

When $\left.s|\geq \delta, \dot{s}=-\varepsilon| s\right|^{\alpha} \operatorname{sign}(s)-k s$

$$
\begin{aligned}
\dot{V}(\mathrm{x}) & =s \dot{s}=s\left(-\varepsilon|s|^{\alpha} \operatorname{sign}(s)-k s\right) \\
& =-\varepsilon|s|^{\alpha+1}-k s^{2}<0
\end{aligned}
$$

When $s k \delta, s k \delta, \dot{s}=-\varepsilon \frac{s}{\delta^{1-\alpha}}-k s$

$$
\begin{gathered}
\dot{V}(\mathrm{x})=s \dot{s}=s\left(-\varepsilon \frac{s}{\delta^{1-\alpha}}-k s\right) \\
=-\left(\frac{\varepsilon}{\delta^{1-\alpha}}+k\right) s^{2} \leq 0
\end{gathered}
$$

It can be seen that formula (4) (5) are all negative, in order to prove that system is asymptotically stable, accord with the requirement of design.

\section{Performance analysis of the control system}

Buck converter initial state $\mathrm{x}_{1}>0, \mathrm{~s}>0$, says the system state moving point at the side of the switching surface $\mathrm{s}>0$.

When $|s| \geq \delta$, the sliding mode reaching law:

$$
\dot{s}=-\varepsilon|s|^{\alpha} \operatorname{sign}(s)-k s<0
$$

At this time, reaching law $s(t)$ are represented by decreasing function, characterized by the sliding mode variable structure control system in the moving point towards the switching surface movement process.

$\varepsilon$ stands for constant speed reaching law among them. When $|s|$ turns larger, if $|s|^{\alpha}>1$, the constant speed reaching law turns enhance. System moving point deviates from the switching surface. The larger $|s|^{\alpha}$ was, the greater the constant speed reaching was. Moving point on the reaching phase 
of reaching the faster the speed, to improve the response speed and anti-interference ability of the system.

When moving point approaches the switching surface $0<s<\delta$, gets the sliding mode reaching law:

$$
\dot{s}=-\varepsilon \frac{1}{\delta^{1-\alpha}} s-k s<0
$$

At this time $s(t)$ still stands for decreasing function, system moving point on the switching surface movement, just on the stage, decreases the speed of the changes.

After selecting constant $\alpha, \delta$, it is not difficult to verify:

$$
0<\frac{1}{\delta^{1-\alpha}}<1
$$

Therefore, when the system moving point reached the switching surface, the role of the constant speed reaching law part of the sliding mode motion compared to conventional exponential reaching law has certain weakening, keeps moving point through switching surface in the low speed, avoiding the moving point through the switching surface at the rate of large and causing the system output strong chattering. At the same time, the figure 2 shows that in the stage of the approach $0<s<\delta$, compared to the change of the exponential function symbol switching function and the saturation function to smooth, comply with the above analysis.

Due to the role of all levels of organization inertia, the system moves point through the switching surface for the first time, gets the sliding mode reaching law:

$$
\dot{s}=-\varepsilon \frac{1}{\delta^{1-\alpha}} s-k s>0
$$

$s(t)$ turns decreasing function to increasing function, prompts system moving point to the switching surface movement. At the same time, because of the system of inertia, moving point moves through the switching surface on stage again. The system of motion control will be adjusted according to the motion law of motion again.

\section{The simulation verification}

Buck converter parameters: $\mathrm{v}_{\mathrm{i}}=20 \mathrm{~V}, \mathrm{~L}=0.00002 \mathrm{H}, \mathrm{C}=0.00015 \mathrm{~F}, \mathrm{R}_{\mathrm{L}}=10 \Omega$. Using exponential reaching law, adopts the sliding mode controller parameters: $c=500, k=3000, \varepsilon=20$. Using the exponential function index reaching law, adopts the sliding mode controller parameters: $\mathrm{c}=500$, $\mathrm{k}=3000, \varepsilon=20, \alpha=0.5, \delta=0.1$.

The system under normal conditions outputs the graphics for the figure 3 . At the same time, in order to verify the exponential reaching law adopts the exponential function and the robustness of system when the load mutation is better than the exponential reaching law. When the time is $0.04 \mathrm{~s}$, the system load mutation is $4 \Omega$, can get the system output graphics as shown in figure 4 .

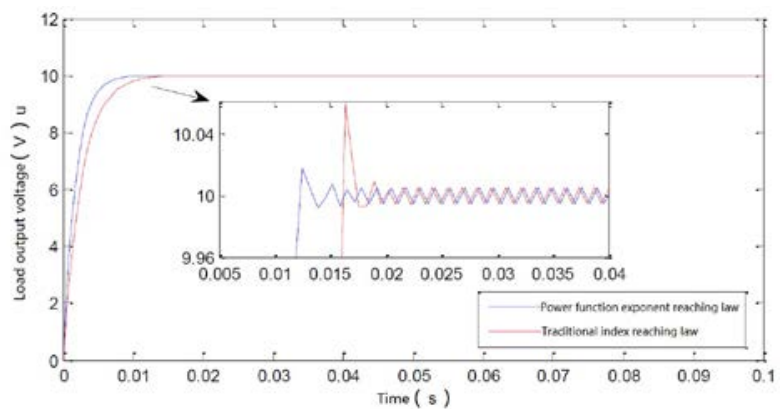

(a) Output voltage comparison

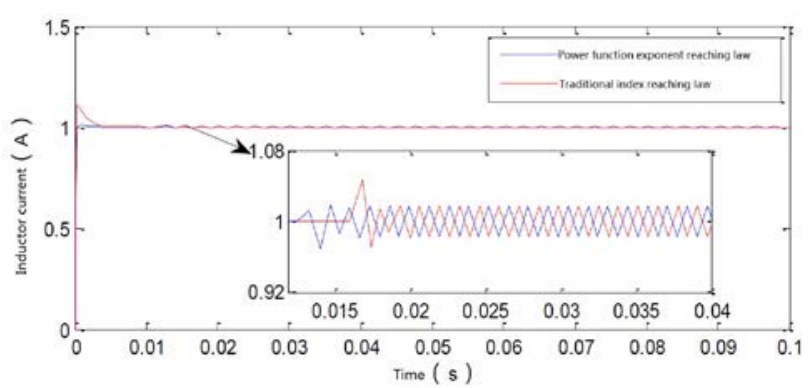

(b) Output current comparison

Fig. 3 The output of the simulation under normal conditions 


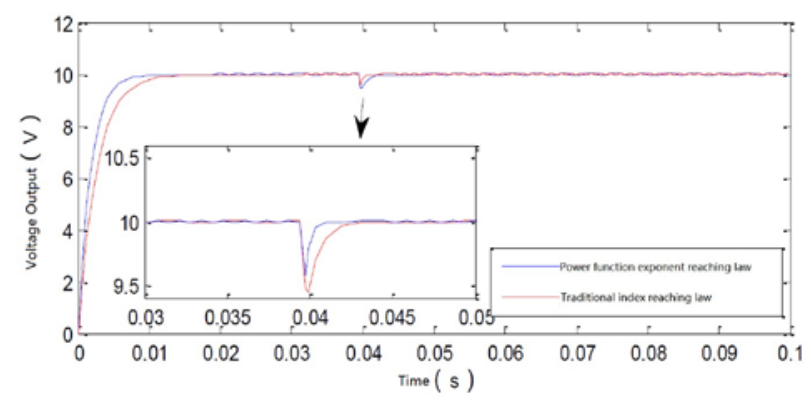

(a) Output voltage comparison

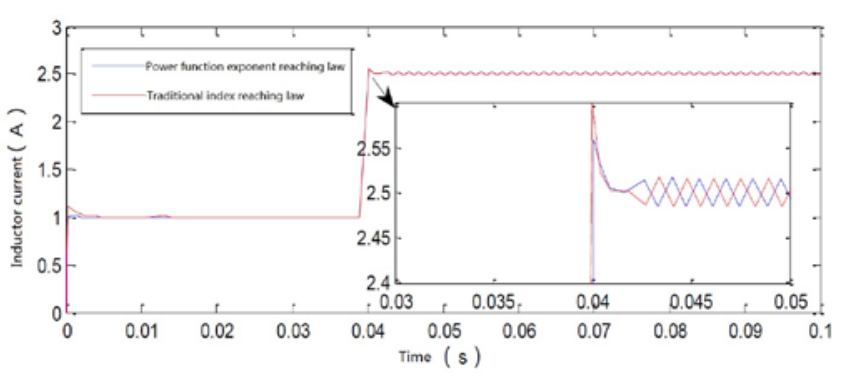

(b) Output current comparison

Fig.4 Output simulation when the load is mutation

Compared with the sliding mode variable structure controller based on traditional exponential reaching law control system, we can see from the figure 3: the arrival time is shorter, shortened from $0.016 \mathrm{~s}$ to $0.016 \mathrm{~s}$ or so, the system overshoot amount.

We can see from the figure 4, when loads become mutton, the system will create a jitter at the instant of the load changing, and then return to the original steady state value. The difference is that, compared with the sliding mode variable structure controller based on traditional exponential reaching law control system, the exponential function based on exponential reaching law of sliding mode variable structure controller under the control of system in the mutation of smaller overshoot and return to the original steady state. Recovery time is shorter. When the load changes, the robustness of the system become better.

\section{Conclusion}

This paper designs a new type of power function index reaching law. It applies to the Buck converter. The reaching law control method is compared with the traditional index, effective shorten the system arrival time and reduce the overshoot of the system. When load becomes mutation, the system shows good robustness. The method can improve system dynamic quality, have certain reference significance for the engineering practice.

\section{Reference}

[1] Gao Weibing. Variable Structure Control Theory Foundation [M]. Beijing: China Science and Technology Press, 1990, p.14-18.

[2] Song Wenzhong, Wen Hong, Yao Qiongkuai. The Discrete Variable Structure Control System of Variable Speed Reaching Law [J], Journal of Marine Engineering Institute, 1999, 28(3) p.16-21

[3] K. Jiang, J.G. Zhang, Z.M Chen. A New Approach for the Sliding Mode Control Based on Fuzzy Reaching Law[C]. Shanghai, China: Proceedings of the 4th World Congress on Intelligent Control and Automation, 2002, p. 656-660.

[4] Luo Cheng. Application Research for Buck Converter on Variable Structure Control [D]. Hubei University of Technology, 2008

[5] Dai Lu. The Control of Sliding Mode Variable Structure on Buck Converter [D]. Northeastern University. 2008.

[6] Zhang Hexin, Fan Jinsuo. A New Double Power Reaching Law for Sliding Mode Control [J]. Control and Decision. 2013, 29(3) p.1-6.

[7] J.X. Xu, Y. J. Pan. T. H.LEE. A Gain Scheduled Sliding Mode Control Scheme Using Filtering Techniques with Applications to Multi-link Robotic Manipulators [J]. Journal of Dynamic Systems Measurement and Control, 2000, 122(4) p.641-649. 
[8] K. Y. Zhuang, H. Y. Su, J. Chu, K. Q. Zhang. Globally Stable Robust Tracking of Uncertain Systems Via Fuzzy Integral Sliding Mode Control [C]. Proceedings of the 3th World Congress on Intelligent Control and Automation, China, 2000, p.1827-1831. 